\title{
Digestive System Neuroendocrine Neoplasm
}

National Cancer Institute

\section{Source}

National Cancer Institute. Digestive System Neuroendocrine Neoplasm. NCI Thesaurus.

Code C27721.

A neoplasm with neuroendocrine differentiation arising from the digestive system. It includes neuroendocrine tumors (well-differentiated endocrine tumors or carcinoid tumors and well differentiated endocrine carcinomas) and neuroendocrine carcinomas (poorly differentiated neuroendocrine carcinomas, small cell carcinomas, and large cell neuroendocrine carcinomas). 